Journal of Applied Fluid Mechanics, Vol. 14, No. 6, pp. 1691-1704, 2021. Available online at www.jafmonline.net, ISSN 1735-3572, EISSN 1735-3645. https://doi.org/10.47176/jafm.14.06.32577

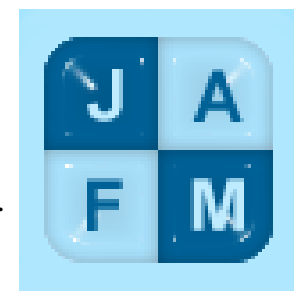

\title{
Mechanism of Stability Enhancement with Shallow Reversed Slot-Type Casing Treatment in a Transonic Compressor
}

\author{
Z. D. Chi, W. L. Chu ${ }^{\dagger}$ and H. G. Zhang \\ School of Power and Energy, Northwestern Polytechnical University, Xi'an 710072, China \\ †Corresponding Author Email: wlchu@nwpu.edu.cn
}

(Received January 14, 2021; accepted May 10, 2021)

\begin{abstract}
In this paper, the influence of a shallow reversed slot-type casing treatment on the performance of a tip-critical transonic compressor has been numerically investigated. Firstly, the complex flow fields in the rotor tip region are studied in details. It shows the severe blockage induced by suction surface boundary separation triggers compressor stall at $100 \%$ design speed, while the blockage due to tip leakage vortex dominates at $80 \%$ design speed. Secondly, the mechanism of stability extension is presented at different rotating speeds. The casing treatment alleviates greatly the tip blockage by manipulating the tip leakage flow, accompanied by the redistribution of aerodynamic loading and mass flux. As a result, the casing treatment is more efficient for the blockage induced by tip leakage vortex (at $80 \%$ design speed). Further analysis of the pressure field and passage shock distribution demonstrates that the passage shock intensity and its location will affect the effectiveness of casing treatment. Finally, the instability characteristics of compressor with casing treatment are revealed. The numerical results reflect when the mass flow approaching the instability boundary, the stator passage blockage presumably is dominant for triggering the compressor stall.
\end{abstract}

Keywords: Axial compressor; Casing treatment; Numerical simulation; Tip leakage flow; Stall margin.

\section{NOMENCLATURE}

$\begin{array}{llll}C_{a x} & \text { axial tip chord } & S C N S & \text { Smooth Casing Near Stall Condition } \\ C T & \text { casing treatment } & S M I & \text { Stall Margin Improvement } \\ C T N S & \text { Casing Treatment Near Stall Condition } & T E & \text { Trailing Edge } \\ G_{s l o t} & \text { normalized mass flow through slots } & V_{z} & \text { axial velocity component } \\ H_{u} & \text { aerodynamic loading coefficient } & V_{r} & \text { radial velocity component } \\ L E & \text { leading edge } & y^{+} & \text {non-dimensional distance from wall } \\ N S & \text { near stall } & U_{t i p} & \text { tip tangential velocity } \\ n_{c o r} & \text { corrected rotating speed } & \phi & \text { normalized mass flow rate } \\ P E I & \text { Peak Efficiency Improvement } & \eta^{*} & \text { adiabatic efficiency } \\ p & \text { static pressure } & \pi^{*} & \text { total pressure ratio } \\ S C & \text { smooth casing } & \rho & \text { density }\end{array}$

\section{INTRODUCTION}

With the increase of stage loading and pressure ratio of modern high-performance transonic compressor, the aerodynamic stability problems such as rotating stall and surge become increasingly prominent. Therefore, it is very important and urgent to take

effective strategies to improve compressor stability (Day 2016; Sun et al. 2019). Casing treatment (CT) has been proved to be an effective passive control method for expanding compressor stability since its discovery (Koch 1970). After years of development, various types of CT have come to the fore, such as groove-type (Houghton and Day 2012; Heinrich et al. 2020), slot-type (Wilke et al. 2005; Alone et al. 
2016), tip injection (Suder et al. 2001; Li et al. 2019), and self-recirculation CT (Hathaway 2002).

Among numerous methods of stability extension, groove-type and slot-type casing treatments have been extensively investigated ( $\mathrm{Lu}$ et al. 2006; Hembera et al. 2008; Du et al. 2017; Zhang et al. 2020), even been applied in real aero-engines because of their effectiveness (Madden et al. 2005). Fujita and Takata (1984) tested a subsonic isolated rotor with varied CT configurations, they found the better the stability extension effect, the more compressor efficiency loss with CT, that is to say, a compromise of stall margin improvement and efficiency loss must be taken into consideration in designing CT. Compared with circumferential grooves, slot-type CT is particularly effective in enhancing the stall margin, so a large number of studies have been conducted recently.

In the early days, only the effect of stability extension could be distinguished due to relatively simple test equipment. As a result, it was difficult to understand the flow mechanisms in-depth. With rapid development of computational fluid dynamics (CFD) and visualization measurement technology, researches have a deeper acquaintance of the stability enhancement mechanism of slot-type CT. In particular, the influence of shock and tip leakage vortex (TLV) on CT effectiveness in transonic compressor has attracted much attention. Hembera et al. (2008) implemented detailed time-accurate simulations in one and half compressor stage with slot-type CT. They found there was a significant difference in the flow phenomenon inside slots due to the interaction of the shock with the pressure distribution of inlet guide vane. Experimental and numerical studies by Streit et al. (2013), using a semi-circle slot-type CT, pointed out that the main function of the CT was to draw off stagnation fluid and improved flow fields by removing the passage blockage induced by TLV. Particle Image Velocimetry (PIV) measurements taken by Brandstetter et al. (2015) also showed the CT could affect the blockage zone, secondary flow and shock structures in the blade tip region. Alone et al. (2016) presented the experimental results of transonic compressor subjected to various configurations of bend skewed slot-type CT. They used the hot wire sensor to capture the oscillations in the inlet axial and tangential velocities, and emphasized that the reduction of oscillation extents is the key role of CT in stability extension. In their study, it was also observed that CT effectiveness differed at different operating speed. The effect of stability extension with CT was poor at design speed, while better at offdesign speed. Zhou et al. (2017) reported the effect of axial position of the slot-type CT on a transonic compressor rotor. They considered that the optimal axial position of CT was closely related to the initial position of the tip leakage vortex and passage shock wave. When the slots covered the tip leakage vortex and the boundary layer separation zone, the CT would lead to largest stall margin improvement.

Summarizing the previous results, we can conclude: (1) Most of works only focused on the stability enhancement mechanism of CT at design speed, the research at off-design speed was rare. From the view of real application, existing studies were too ideal and only biased in laboratory conditions without considering the effects of real geometry constraints. (2) Although reviewers gradually realized the influence of shock structures and tip leakage vortex on designing effective $\mathrm{CT}$, further studies were still needed to reveal the interaction between shock wave/tip leakage vortex and shock wave/boundary layer, especially at off-design speed of compressor. Motivated by these questions, the effects of a novel shallow reversed slot-type CT on the performance of a transonic axial compressor were studied through steady and unsteady simulations at design and offdesign speed.

The organization of this paper is as follows: the configurations of compressor stage and SRSCT are described in Section 2, the numerical method and grid is introduced in Section 3. In Section 4, the compressor instability characteristics at different rotating speeds are studied at first. Then, the influence of the pressure field and the shock wave on the stability enhancement of SRSCT is presented. Finally, main conclusions are summarized in Section 5.

\section{COMPRESSOR MODEL AND CASING TREATMENT}

\subsection{Investigated compressor}

A single stage, transonic, high speed axial compressor is used throughout this paper, the configurations of compressor stage can be found in Fig.1. The main design parameters of the compressor are given in Table 1 .

Table 1 Main design parameters of compressor.

\begin{tabular}{|c|c|}
\hline Parameters & values \\
\hline Average shroud diameter(mm) & 200 \\
\hline Average hub diameter(mm) & 120 \\
\hline Tip clearance(mm) & 0.2 \\
\hline Blade tip axial chord(mm) & 50 \\
\hline Rotor blade number & 18 \\
\hline Stator blade number & 41 \\
\hline Mass flow(kg/s) & 20 \\
\hline Design rotating speed(rpm) & 26000 \\
\hline
\end{tabular}

The compressor stage consists of 18 rotor blades and 41 stator blades. Tip clearance of rotor is representative of a modern high speed compressor, the design speed reaches to $26000 \mathrm{rpm}$, with the design flow rate $20 \mathrm{~kg} / \mathrm{s}$ and the total pressure ratio 1.95. Experiments carried out have shown that the compressor is tip-critical stall inception, so it is especially suitable for the application of casing treatment to improve the stability margin (Greitzer $e t$ al. 1979). For figuring out the role and difference of slot-type CT under different speeds, the following is a comparative study of $\mathrm{CT}$ at two different rotational speeds $(100 \%$ and $80 \%)$. 


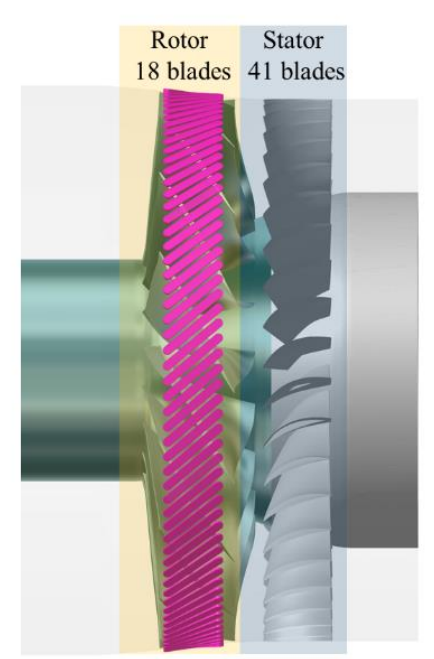

(a)

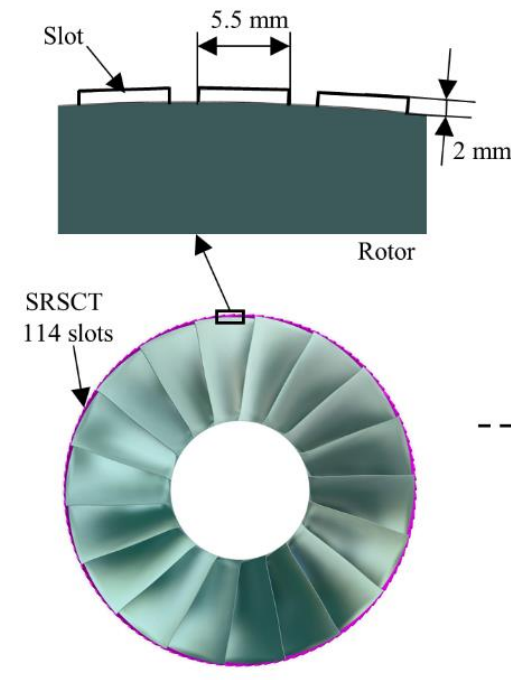

(b)

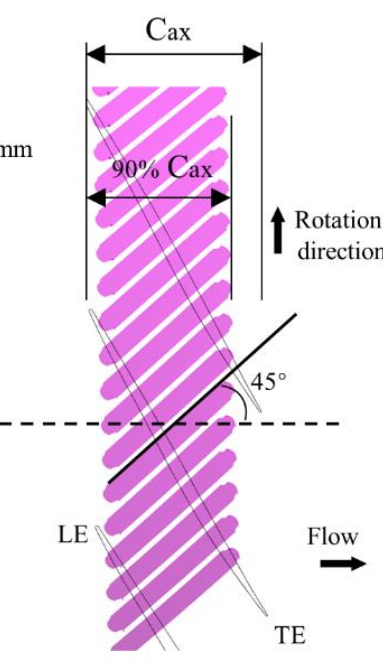

(c)

Fig. 1. Configurations of compressor stage and SRSCT. (a) compressor stage; (b) and (c) SRSCT.

\subsection{Casing treatment configurations}

According to the design experience of traditional casing treatment parameters (Wilke and Kau 2004 and Gelmedov et al. 2014), the slot depth of casing treatment is about 3 times the slot width. On the basis of the author's knowledge, the traditional casing treatment design does not fully consider the constraints of real compressor geometric structure, so many problems will be encountered in practical applications of casing treatment. In this article, we design a novel shallow reversed slot-type casing treatment considering the limitation of the outer wall of the investigated rotor casing, the slot depth (about $2 \mathrm{~mm}$ ) is only one third of slot width, and the axial direction is reverse to blade stagger angle, so we named it shallow reversed slot-type casing treatment (SRSCT).

The configurations of SRSCT are illustrated in Fig. 1(b) and Fig.1(c).

The structure of SRSCT is straight slot with rounded end, opposite to the stagger angle of rotor blade, and about 45 degrees with axial direction. Detailed parameter values of SRSCT are following: the axial length of slot is equal to $90 \%$ blade tip axial chord $\left(C_{a x}\right)$ and the starting location of the casing treatment is from the leading edge of rotor, the slot depth is only $2 \mathrm{~mm}$, the slot width is about $5.5 \mathrm{~mm}$, the slot number for whole annular is 114 , the open area ratio of SRSCT is up to $66.7 \%$. Generally, it is known that smaller amount of overlap of casing treatment is beneficial for reducing efficiency loss, while this paper mainly focuses on the difference of casing treatment effect under different rotational speeds, so a compromise design is adopted for the parameters of SRSCT, that is to say, it isn't considered with the optimization of the casing treatment structure in present research.

\section{NUMERICAL METHOD AND VALIDATION}

\subsection{Numerical method}

The present simulations are performed by commercial CFD solver, NUMECA (2011). The 3D Reynolds averaged Navier-Stokes $(R A N S)$ equations under the relative frame of reference are discretized in space with a cell-centered finite volume formulation, and the one equation S-A turbulence model is applied. To save computing time, the computational domain is reduced to $3: 7$ meshed passages.

For the steady simulations, the explicit four-step Runge-Kutta time marching method is used to obtain the steady-state results, and the methods of multigrid, local time step and implicit residual smoothing are used to speed up convergence. For the unsteady simulations, the implicit dual time stepping method is used to solve the equations. To capture the unsteady flow phenomenon sufficiently, the physical time step is about $2.5 \times 10^{-6} \mathrm{~s}$, which represents the physical time step is 60 in one rotor blade pitch. At each physical time step, the number of the virtual time step for inner iteration is 30. In steady simulations, the frozen rotor schemes are applied on the R1/S1 interface and full non-matching are used at the interface R1 and SRSCT. For unsteady simulations, the domain scaling method is imposed on the R-S interfaces.

During the numerical simulation, adiabatic and noslip boundary conditions are applied at all solid walls. Inlet total pressure, total temperature and axial flow condition are set at the inlet boundary. Static back pressure is set at the outlet boundary, and simple radial equilibrium equation is used to get the outlet static pressure distribution. Back pressure is 
increased gradually to get stable numerical solutions, at near stall condition, the back pressure is raised by $100 \mathrm{~Pa}$ (both steady and unsteady simulation) increments to obtain the last convergent solution.

The grids in main flow passages and slots of SRSCT are generated by IGG/Autogrid5, the computational grid of compressor stage and SRSCT are shown in Fig. 2 in details. The rotor and stator blade passages are meshed in H-O-H topology, which consists of a main O-block surrounding the blade, H-block generated in the inlet and outlet domains. Additionally, butterfly grid topology is embedded into the tip gap. The grid density was increased towards the solid boundaries to keep $y^{+}<2$ at the walls for accurately capturing the viscous fluxing. Grids in three rotor passages amount to 2.6 million and 7 stator passages 2.8 million, single passage mesh generated first and duplicated to others. For the SRSCT grid setting, the middle horizontal segment adopts the simple H-block topology, and butterflygrid topology at both ends of the arc segment. The grids in single slot consist of 49 nodes in axial direction, 17 nodes in the span-wise direction and 17 nodes in circumference direction.

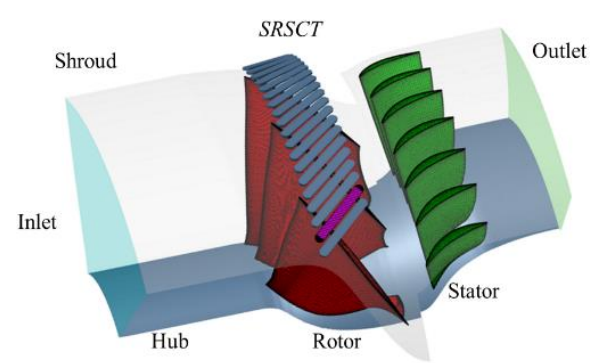

(a) 3D diagram of computational domain

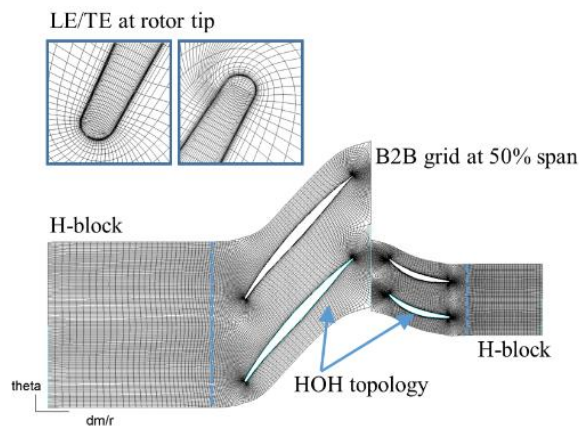

(b) B2B grid configuration at rotor middle and tip

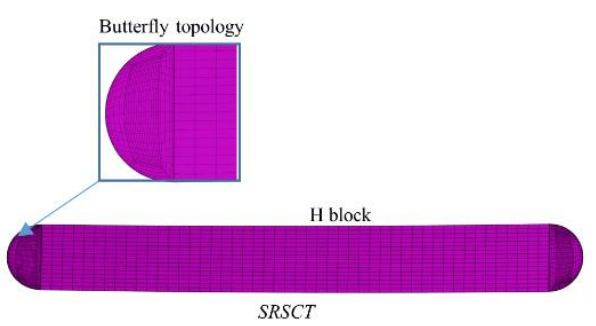

(c) Grid configuration of SRSCT

Fig. 2. Computational grid of compressor stage and SRSCT.
The number of total slot grids up to 1.04 million, so the computational model with casing treatment comprises of 6.4 million nodes.

\subsection{Numerical validation}

In order to verify the accuracy of the numerical method, the NASA stage 35 is used to validate the numerical model mentioned above. NASA stage 35 is one of the low aspect ratio transonic inlet stages, designed by NASA Lewis research center in 1978 to verify the influence of aspect ratio and load on compressor performance. The compressor consists of 36 rotors and 46 stators, the design rotational speed is $17188 \mathrm{rpm}$, with the stage total pressure ratio 1.82 at design mass flow $20.18 \mathrm{~kg} / \mathrm{s}$. NASA stage 35 has high similarity in the loading and critical flow phenomenon at the rotor tip with the compressor in this paper. According to Reid and Moore (1978) and Chima (2009), the rotor clearance is set $0.4 \mathrm{~mm}$ and the stator gap is set $0.76 \mathrm{~mm}$ separately, and the numerical performance acquisition points are consistent with experiments.

Figure 3 presents the comparison of stage performance at $100 \%$ design speed with 3 different grids number symbolled as G1, G2, G3 (about 0.8 , 1.3 , and 2 million cells) per passage, the symbols $\phi, \pi^{*}$ and $\eta^{*}$ represent the normalized mass flow rate, total pressure ratio and adiabatic efficiency respectively. All three grids predict results are consistent with the experimental data, the difference exists only at the near stall condition. As the grid number increase, the near stall mass flow is close to $\phi=0.92$, it shows that the level G2 has met the need of simulation accuracy. Note that the near stall point did not reach the experimental value, the report by Reid given the stall point of Stage 35 as $\phi=0.87$. As stated by Chima (2009), the rotor is probably in stable rotating stall at this operating point, so a steady simulation hardly reached this point.

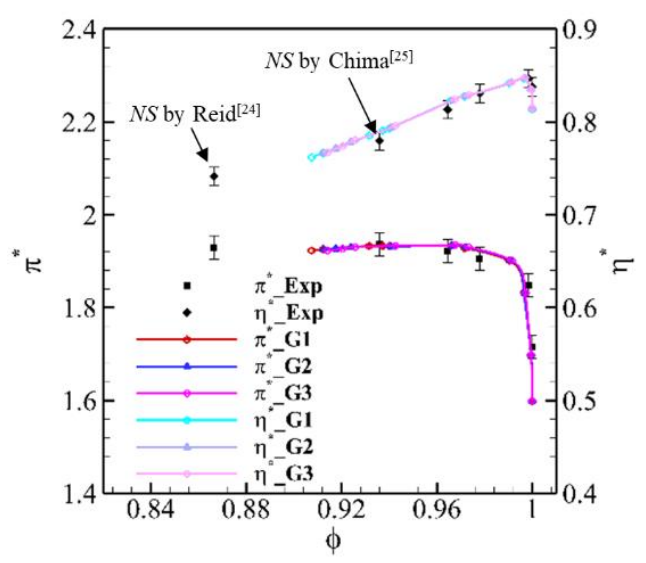

Fig. 3. Performance maps of stage 35 at $100 \%$ design speed.

Span-wise distributions of total pressure downstream of the rotor are compared to experimental data in Fig. 4. Two operating points shown are peak efficiency 
$(P E)$ point and near stall $(N S)$ point. Computed total pressure profiles agree fairly well with the data above 0.35 span, but the predicated value was higher at $P E$ while lower at $N S$, which is agreement with compressor characteristics lines. Note that rotor 35 has a region of lower total pressure below 0.35 span that is not quite captured by the calculations, which might be due to the numerical method or real hub leakage.

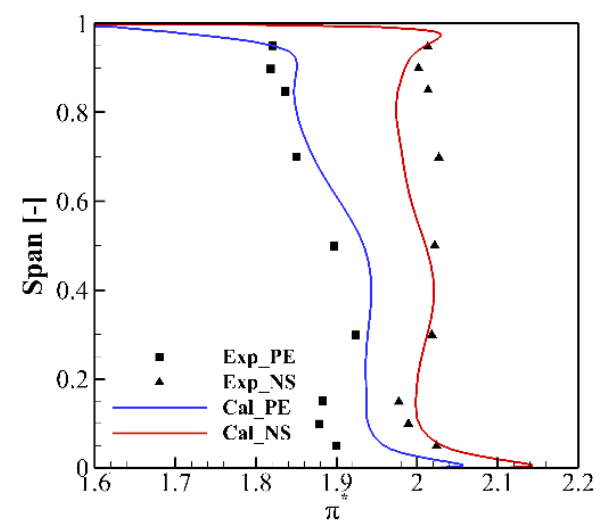

Fig. 4. Span-wise distributions of total pressure ratio at $P E$ and $N S$ condition

\section{RESULTS AND DISCUSSIONS}

\subsection{Compressor overall performance}

The performance maps of the compressor with and without SRSCT at two rotating speeds $\left(n_{c o r}=1.0\right.$ and $\left.n_{c o r}=0.8\right)$ are shown in Fig.5. In the Figure, different solid lines (identified by SC $n_{\text {cor }}=1.0$, CT $n_{\text {cor }}=1.0$ etc.) represent the steady simulation results, and the discrete solid points (identified by SC $n_{\text {cor }}=1.0$ un, $\mathrm{CT} n_{c o r}=1.0$ un etc.) present the unsteady simulation results. Arrows labeled with "SCNS" mean the NS condition with smooth casing $(S C)$, the arrows labeled with "CTNS1" and "CTNS2" represent two NS conditions (their mass flow is close) with SRSCT. The symbol $\phi$ represents mass flow, which is normalized by choked mass flow with SC.

The stall margin improvement (SMI) and peak efficiency improvement (PEI) are defined to quantitatively analyze the effect of SRSCT on the compressor overall performance, and it can be derived from following equations:

$$
\begin{aligned}
& S M I=\left[\frac{\pi_{C T}^{*}}{\pi_{S C}^{*}} \cdot \frac{\phi_{S C}}{\phi_{C T}}-1\right] \times 100 \% \\
& P E I=\left[\frac{\eta_{C T}^{*}}{\eta_{S C}^{*}}-1\right] \times 100 \%
\end{aligned}
$$

where $C T$ and $S C$ represented casing treatment and smooth casing condition, $\pi^{*}, \phi$ and $\eta^{*}$ represented total pressure ratio, mass flow and peak efficiency, respectively.

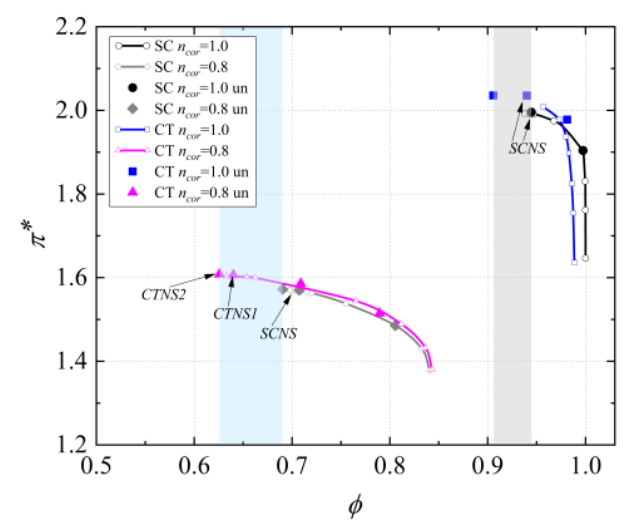

(a) Total pressure ratio

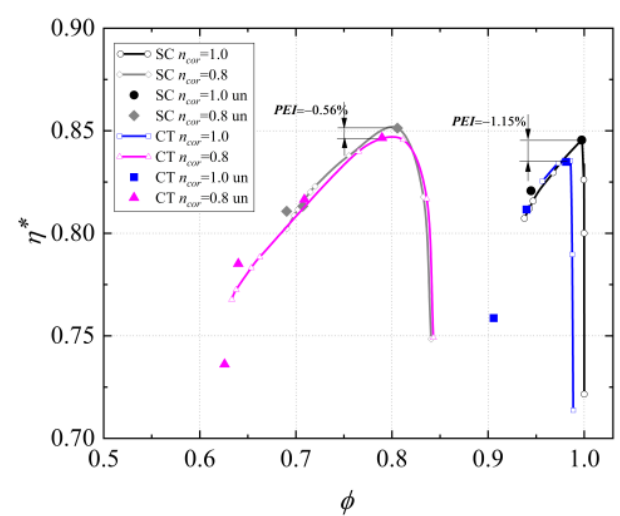

(b) Adiabatic efficiency

Fig. 5. Performance maps of compressor with and without SRSCT at different speeds.

At $100 \%$ design speed, the SRSCT does not show the effect of stability enhancement by steady calculation results, while it brings about $S M I$ to $8.7 \%$ and $P E I$ to $-1.15 \%$ by unsteady calculation results. It shows the steady calculation exists some shortcomings in the evaluation of stability enhancement of $C T$. By contrast, great stability margin enhancement is obtained at $80 \%$ design speed. Under unsteady calculations, the SMI and PEI calculated are $15.6 \%$ and $-0.56 \%$ at $80 \%$ design speed.

Visualization flow fields analysis will be carried out below to provide further demonstration on the instability flow characteristic for smooth casing and applied SRSCT. Then, the mechanism of stability enhancement with SRSCT will be revealed by analyzing the unsteady results in details. Besides, the difference in SMI at two speeds is discussed indepth.

\subsection{Instability characteristics under smooth casing}

According to the flow characteristics in compressor tip region, Wilke et al. (2005) divided the compressor spike stall into blade tip stall and tip blockage stall. Generally, blade tip stall (Wang et al. 2020), is caused by the compressor blade aerodynamic overload, which leads to a large range of flow separation on the suction surface of the blade. Tip blockage stall (Furukawa et al. 1998; Hofmann 
et al. 2002), however, is usually induced by tip leakage vortex. In contrast to blade tip stall, the tip blockage stall mechanism is more common in transonic compressor and more suitable for CT to improve the stall margin of compressor (Wilke et al. 2005; Hembera et al. 2008).

In this paper, the parameters defined by Wilke $e t$ al. (2005) are used to figure out the critical flow state in present compressor. As stated by Wilke, it can be simply decided whether the primary stall mechanism is blade tip stall or tip blockage stall. The parameter $B_{p}$ and $B_{s}$ are defined as follows:

$$
\begin{aligned}
& B_{P}=\frac{\gamma-\xi}{\chi-\xi} \\
& B_{S}= \\
& M_{1, P} \tan (90-\beta) \cos (180-(\gamma+\beta)) / M_{2, P}
\end{aligned}
$$

\section{Relative Mach Number}

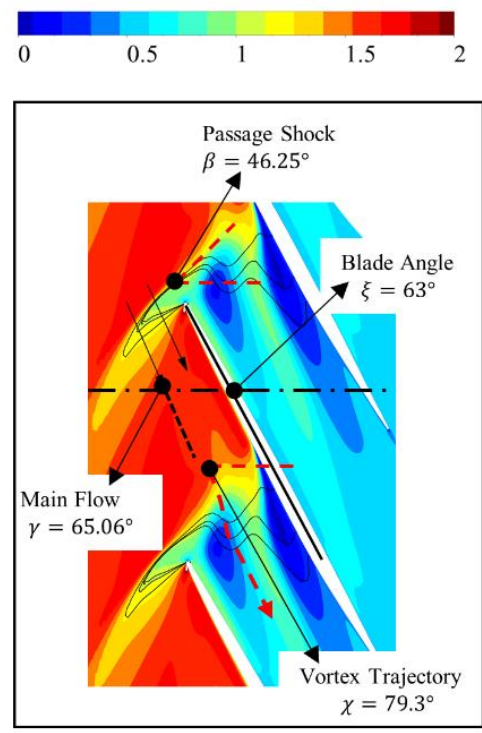

(a) $n_{\text {cor }}=1.0$ where $\gamma, \beta, \xi$, and $\chi$ represents the angle parameter in the flow field, $M_{1, P}$ and $M_{2, P}$ represents the perpendicular component upstream and downstream of the passage shock, more details can be found in Hembera et al. (2008).

Figure 6 shows the flow angles at 0.98 span at $100 \%$ and $80 \%$ design speeds at SCNS point. The contours of relative Mach number are also superimposed in the figure. According to the Eqs. (3) and (4), $B_{P}$ is 0.7 at $100 \%$ design speed. While $B_{P}$ is 0.2 and $B_{S}$ is 0.57 at $80 \%$ design speed. It indicates that the stall mechanism may be different at two speeds, blade tip stall may occur at $100 \%$ design speed while tip blockage stall at $80 \%$ speed. The Mach number distribution shows that the blockage induced by suction surface separation dominates at $100 \%$ design speed while the blockage induced by tip leakage flow dominates at $80 \%$ design speed.

Fig. 6. Flow angles at 0.98 span at different speeds.

\subsection{Mechanism of stability enhancement with SRSCT}

To figure out the the mechanism of stability enhancement with $S R S C T$ at different rotational speeds, the flow fields in the slots of SRSCT and its influence on the blade passage flow will be discussed. The following analysis focus on SCNS condition, i.e., all the results in this section are compared at the same mass flow point.

The discussion starts with features of SCNS condition at $100 \%$ design speed. Figure 7 depicts the time-averaged 3D limiting streamline distributions of TLV, as well as reversed flow region (grey zones) at 0.98 span with and without SRSCT. Meanwhile, in order to describe the influence of SRSCT on shock location and TLV, the region of the dotted box in Fig. 7(a) is enlarged to Fig. 8 , Fig. 8 shows the timeaveraged distributions of static pressure, where the detached shock location is marked with black solid line, and the red dotted line represents the tip leakage vortex trajectory. The static pressure is normalized by inlet total pressure symbolled as $p_{\text {ref }}$.

For $S C$, the tip leakage vortex expands after it cross the passage shock, and induces an obvious revered flow zone near adjacent pressure surface. Moreover, separation induced by shock/boundary layer effect occupies a significant fraction of the tip region. As a result, the blade tip passage blockage is formed by suction surface separation and tip leakage vortex together, which may be the main reason of compressor instability when the compressor is throttled to lower mass flow. 


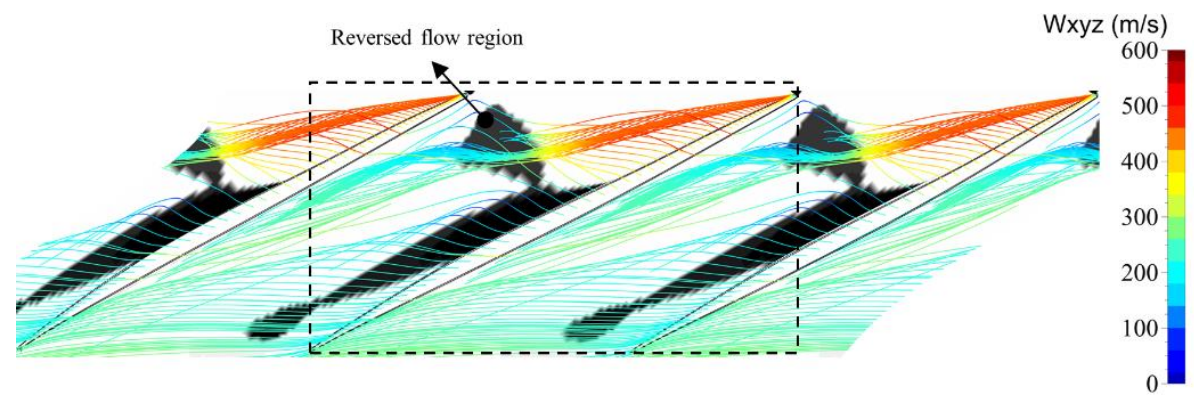

(a) $\mathrm{SC}$

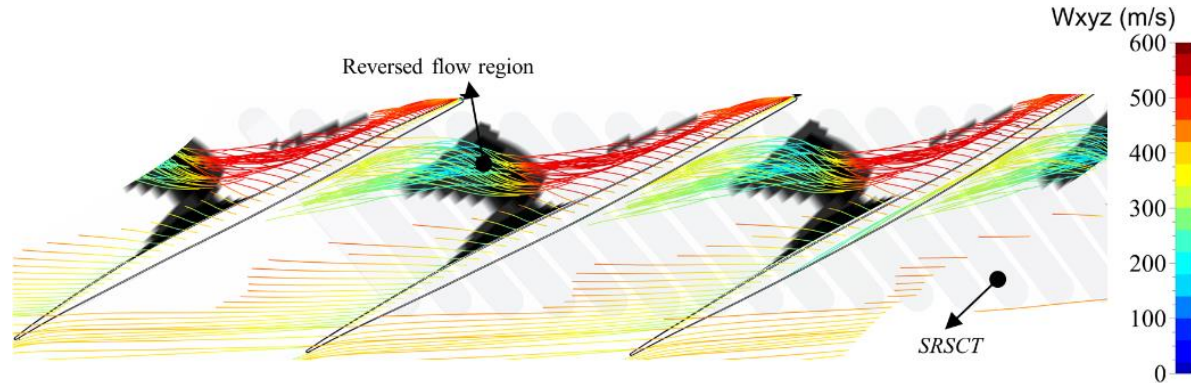

(b) SRSCT

Fig. 7. Time-averaged distributions of tip leakage flow and reversed flow zone on SCNS at $100 \%$ design speed.
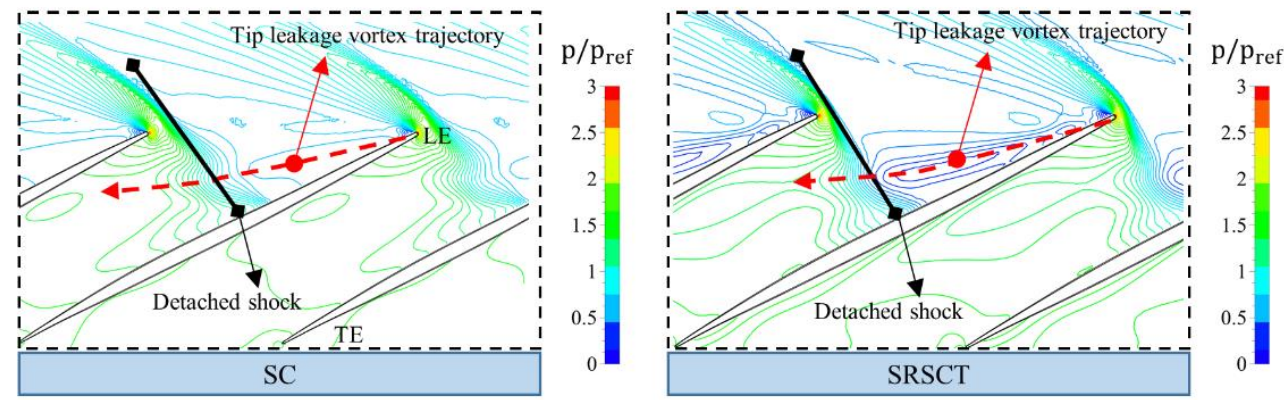

Fig. 8. Time-averaged distributions of static pressure on SCNS at $100 \%$ design speed.

Under the effect of the SRSCT, passage shock moves downstream (right plot in Fig.8) and the tip leakage flow intensity increases, thus the suction surface separation reduces slightly, yet the blockage induced by TLV still occupies most of reversed flow zones. It also can be found the tip leakage flow is sucked into the SRSCT slots, because parts of tip leakage flow streamline vanish. By comparison, the tip leakage vortex trajectory has no significant change. The flow field contrast of SC and SRSCT at $100 \%$ design speed indicates that the effect of SRSCT is limited when the tip blockage induced by suction surface separation.

In contrast, time-averaged distributions of 3D limiting streamline of TLV, as well as reversed flow region (grey zones) at 0.98 span with and without SRSCT at $80 \%$ design speed are shown in Fig. 9, and the time-averaged distributions of static pressure are presented in Fig.10 accordingly. The marks and symbols are consistent with Fig.7 and Fig.8.

Different from $100 \%$ design speed, the tip blockage induced by tip leakage vortex is dominant at $80 \%$ design speed. The TLV cause a large area of blockage, the trajectory of TLV is almost parallel to leading edge of adjacent blade (left plot in Fig.10), and forward spillage occurs. According to the criteria for the spike stall inception proposed by Vo 2008, the spillage of the tip leakage flow is an indicator of spike disturbance.

At $80 \%$ design speed, SRSCT greatly suppresses the blockage induced by TLV, and the shock is pushed further downstream, accompanied by disappeared forward spillage. As a result, the tip passage flow capacity is greatly improved. It can be concluded the manipulation of TLV by SRSCT is the fundamental reason for stability enhancement of compressor, and the SRSCT is more effective when tip blockage is dominated by TLV.

With the aim to reveal the reason for the change of TLV with SRSCT, the changes of mass flux $\left(\rho \cdot V_{Z}\right)$ and aerodynamic loading coefficient $\left(H_{u}\right)$ in the meridian plane for SRSCT at $100 \%$ and $80 \%$ design speed are illustrated in Fig. 11 and Fig. 12, the 


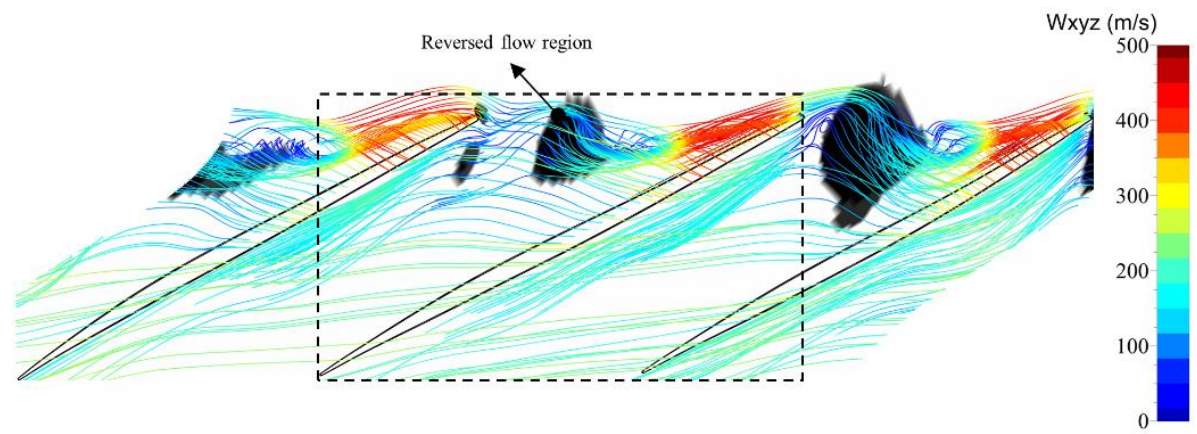

(a) $\mathrm{SC}$

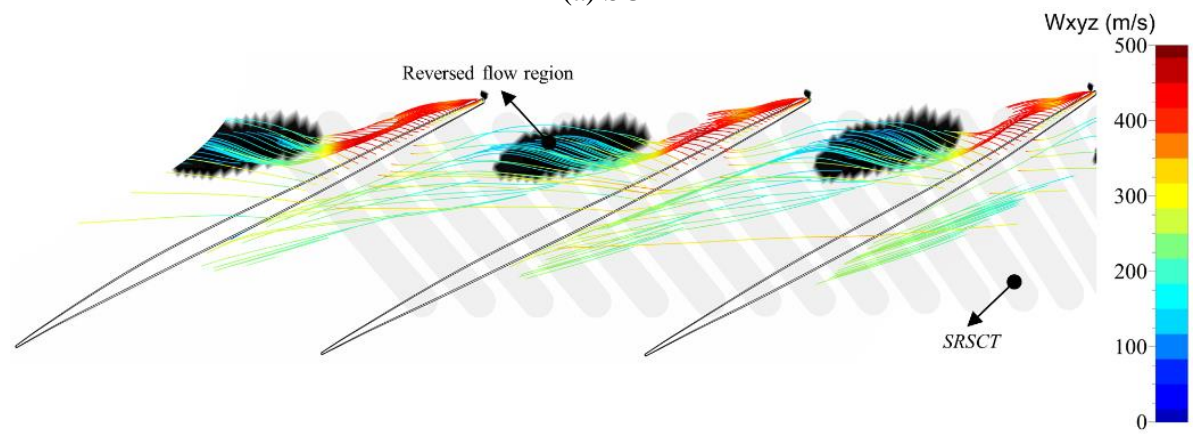

(b) SRSCT

Fig. 9. Time-averaged distributions of tip leakage flow and reversed flow zone on SCNS at $80 \%$ design speed.
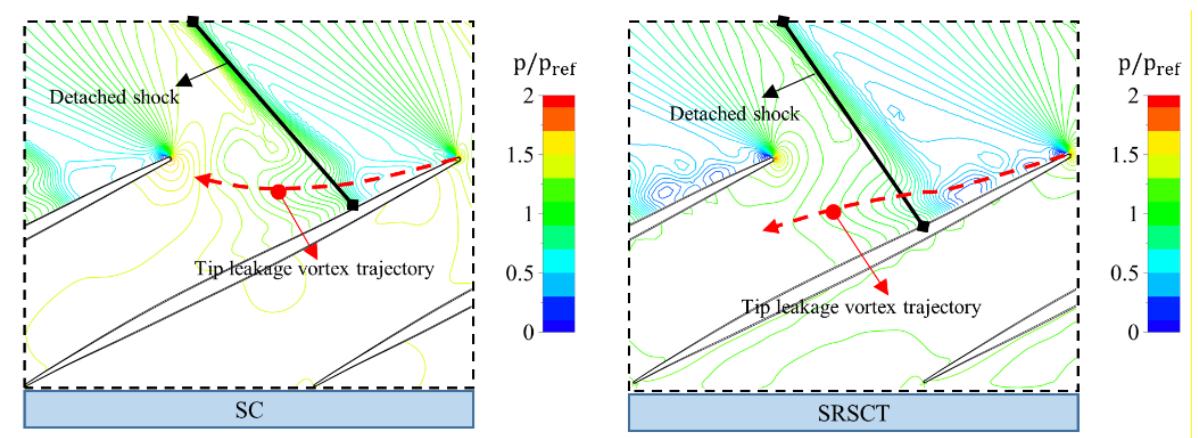

Fig. 10. Time-averaged distributions of static pressure on SCNS at $80 \%$ design speed.

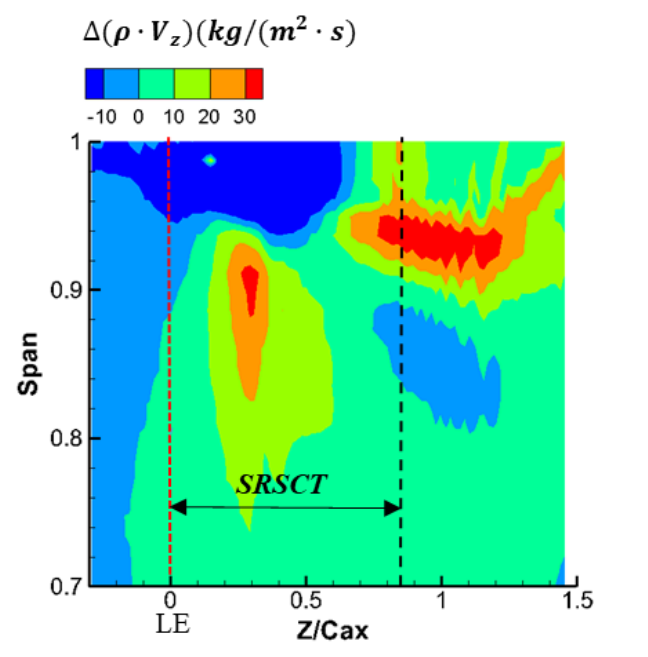

$100 \%$ Design speed

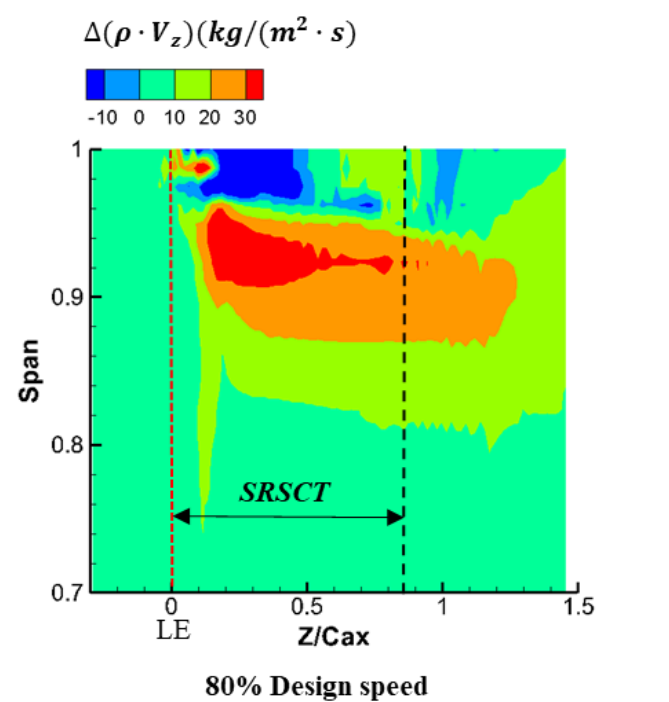

80\% Design speed

Fig. 11. Changes of mass flow in the meridian plane with casing treatment. 

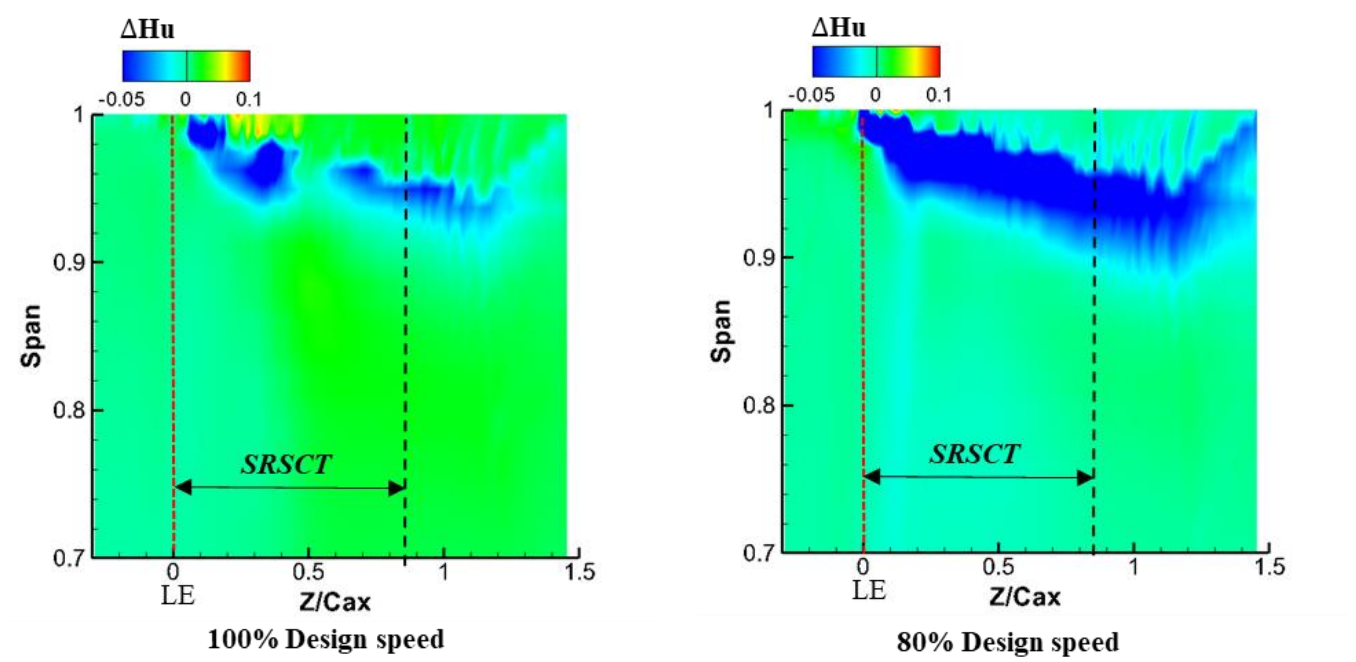

Fig. 12. Changes of aerodynamic loading coefficient in the meridian plane with casing treatment.

abscissa represents the location to the leading edge of rotor and the ordinate represents the span-wise height, only above 0.7 span are shown. The loading coefficient is defined as follows:

$$
\left.H_{\mathrm{u}}=C_{\mathrm{p}} \cdot T_{1}^{*} \cdot \pi^{* \frac{k-1}{\mathrm{k}}}-1\right) / U_{\text {tip }}^{2}
$$

Where $T_{1}^{*}$ represents the inlet total temperature, $\pi^{*}$ represents the total pressure ratio and $U_{\text {tip }}$ is the tip tangential velocity.

In general, the mass flux and aerodynamic loading coefficient are reduced above 0.9 span because of the effect of SRSCT, and the mass flux is increased below 0.9 span, that is, the mass flux is redistributed along the span-wise due to the influence of SRSCT. According to previous studies (Zhang et al. 2020; Wang et al. 2019), the redistribution of mass flux improves the passage flow capacity. As a result, the rotor can bear lower mass flow and higher inflow angle, and the reduced aerodynamic loading leaves more space of achieving higher pressure rise before the rotor runs into stall.

As is evident, the effect of SRSCT is quite different at $100 \%$ and $80 \%$ design speed. At $100 \%$ design speed, the mass flux adequately reduces above 0.95 span at the active region of SRSCT, while the mass flow increases under 0.95 span and rear of casing treatment discontinuously (left plot in Fig.11). Moreover, it can be observed from the left plot in Fig.12 the reduced fraction of aerodynamic loading coefficient is small and interrupted, which results from the effect of the strong passage shock wave.

At $80 \%$ design speed, the changes of mass flux and aerodynamic loading coefficient emerge dramatic difference contrast to that of $100 \%$ design speed. Above 0.95 span, the mass flux is increased at the leading edge of rotor, and the mass flux continuously increases below 0.95 span (right plot in Fig.11). The changed fraction of aerodynamic loading coefficient is stretching across entire above 0.9 span, which indicates the effect of SRSCT on tip loading reduction and mass flux redistribution is remarkable at $80 \%$ design rotational speed. To the author's knowledge, the difference of stability extension effect at different speeds presumably comes from rotor tip pressure field and passage shock wave, so the distributions of static pressure and radial component of absolute velocity will be discussed following.

Subsection summary: (1) The difference of stall margin improvement at different speeds result from the difference of dominant factors causing tip blockage. The SRSCT is more effective when tip blockage is dominated by TLV. (2) The intensity or extent of tip loading reduction and mass flux redistribution can be an indicator of effectiveness of SRSCT.

\subsection{Influence of passage shock on the effectiveness of SRSCT}

For revealing the effect of passage shock intensity and its location on stability enhancement of SRSCT at different speeds, distributions of static pressure and radial component of absolute velocity at 0.98 span are given in Fig. 13 and Fig. 14. In the figures, the symbol $\Delta p$ and $\Delta V_{\mathrm{r}}$ is the difference between SRSCT and SC at SCNS condition, and the $\Delta p$ is normalized by inlet total pressure symbolled as $p_{\text {ref }}$.

With SRSCT, the tip pressure fields have changed significantly. There exists a zone (marked as "change of shock" in Fig.13) which divides the flow fields into two parts. One is the suction effect zone at rear passage and another is the injection effect zone near leading edge. As is evident, the extent of changed shock zone has an obvious influence on the suction effect and injection effect of SRSCT.

At $100 \%$ design speed, the extent of the changed shock zone is bigger and closer to the rear passage, and the suction effect zone and injection effect zone is completely separated. As well known, the 

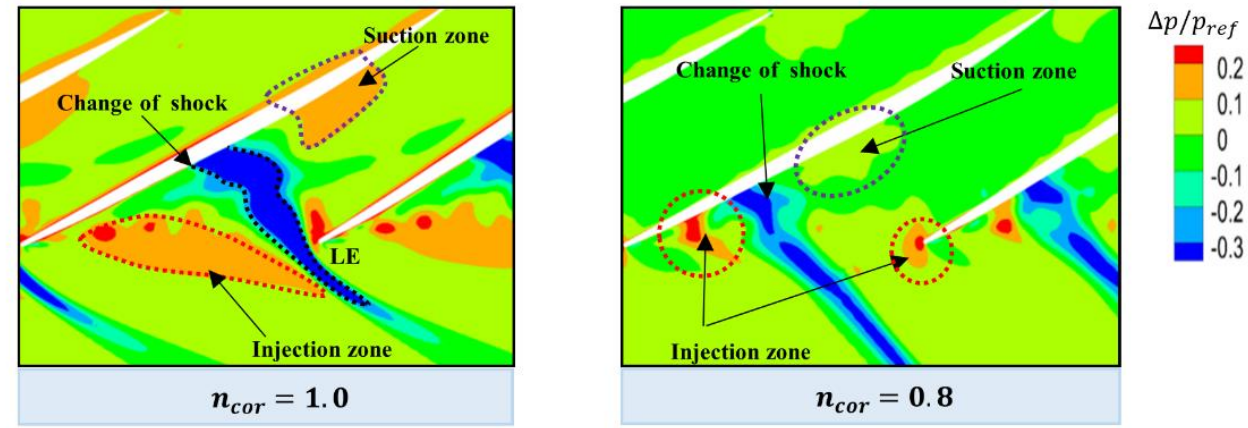

Fig. 13. Changes of static pressure distribution at 0.98 span with casing treatment.
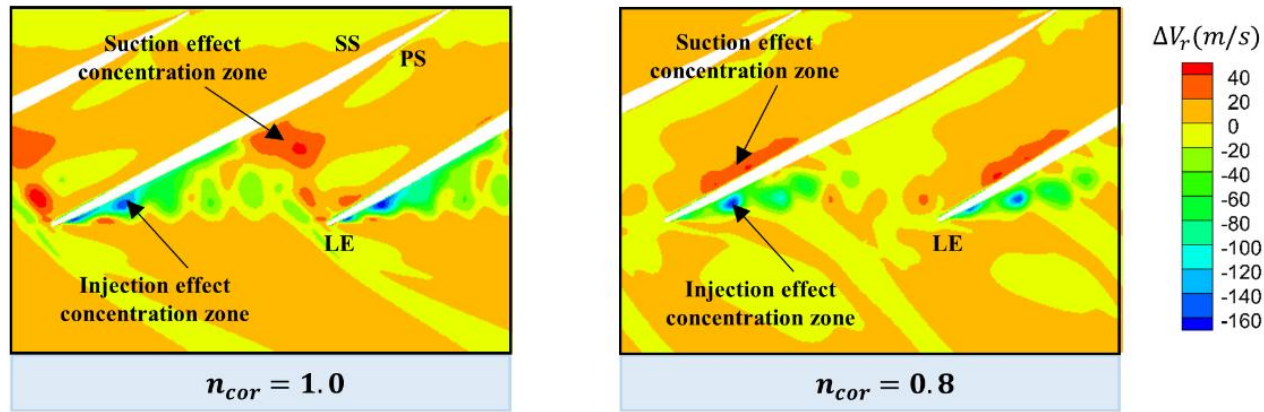

Fig. 14. Changes of Radial velocity distribution at 0.98 span with casing treatment.

difference of static pressure between pressure surface and suction surface is expected to drive the flow circulation of slots in designing CT (Wilke et al. 2005; Lu et al. 2006). At 100\% design speed, however, the shock wave is strong and non-detached, resulting in the suction effect concentration zone being still on blade suction surface side (left plot in Fig.14). As a result, the effective recirculation flow in slots is weaken to some extent, which can also be seen from the Fig.10(b). Therefore, the SRSCT does not bring about sufficient stall improvement because of the influence of passage shock.

Different from $100 \%$ design speed, the shock wave is entirely detached and the intensity of shock wave weakens at $80 \%$ design speed, so the extent of the changed shock zone is also affected. For this reason, the suction effect concentration zone at $80 \%$ design speed is appropriate on blade pressure surface side as expected. Owing to the change of shock wave and pressure fields, the flow recirculation in slots is more effective, so the stability enhancement of SRSCT is significant. Summing up the above analysis, one can conclude that passage shock and its location plays a key role in the effectiveness of SRSCT. When designing $\mathrm{CT}$, a suitable position must be fully considered to ensure the effective flow recirculation in slots.

For an effective circulation of slot-type casing treatment, the rear suction effect and front injection effect are equally important, separate suction effect or injection effect may cause flow recirculation failure in slots. Instantaneous radial velocity distributions on the opening surface of the slots are shown in Fig. 15, for evaluating the flow recirculation intensity in slots, where the radial component of absolute velocity $V_{r}$ is normalized by the rotor blade tip tangential velocity $U_{\text {tip }}$. Symbols "NO.1 to NO.19" represent the different slots and three blade passages are numbered (1), (2), and (3).

At $100 \%$ design speed, the strong passage shock wave steepens the pressure field behind the shock wave. Because of the installation direction of the slot is parallel to the shock wave, as a slot (such as NO.2 slot in Fig. 15) passes through the shock wave, the underneath high pressure field makes the flow in the slot unable to jet into the tip region, so the flow accumulates in the slot. At the time the slot is far away from the shock wave, the delayed injection flow happens. Based on this, we speculate that a fraction of invalid slot flow recirculation influenced by passage shock leads to the insufficient stall margin improvement with SRSCT at $100 \%$ design speed. At $80 \%$ design speed, however, the shock wave is entirely detached and the intensity of shock wave weakens, so the invalid flow recirculation caused by strong shock wave gets relief. As a result, the SRSCT can bring about higher stability margin improvement at $80 \%$ design speed.

The instantaneous normalized mass flow distributions through the opening surface of different slots are depicted in Fig. 16. Values greater than zero represent the flow into the slot, and vice versa. In the Figure, the abscissa represents different slots (not the one or more period), and the ordinate represents normalized mass flow through slot opening surface (the mass flow is normalized by outlet mass flow of compressor at SCNS condition). Different solid lines 


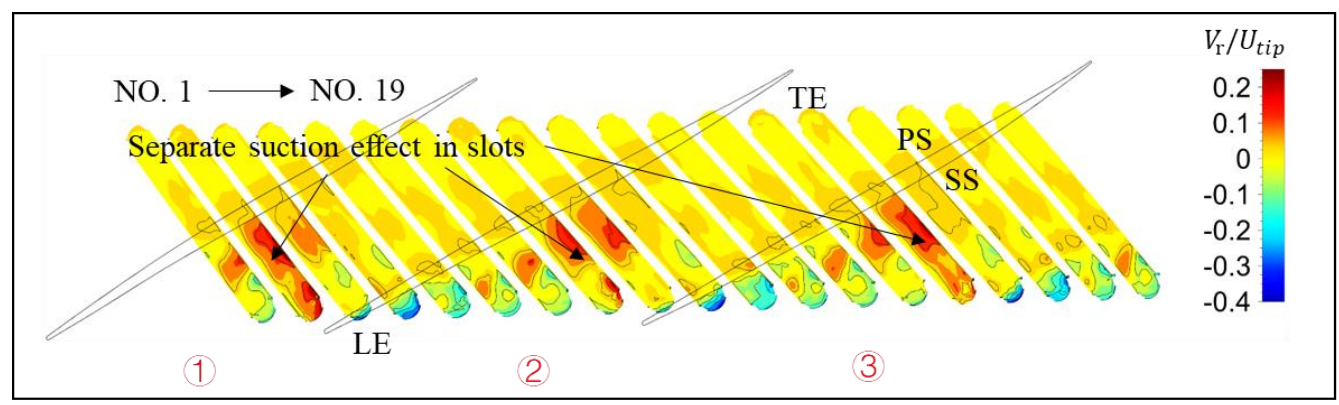

(a) $100 \%$ design speed

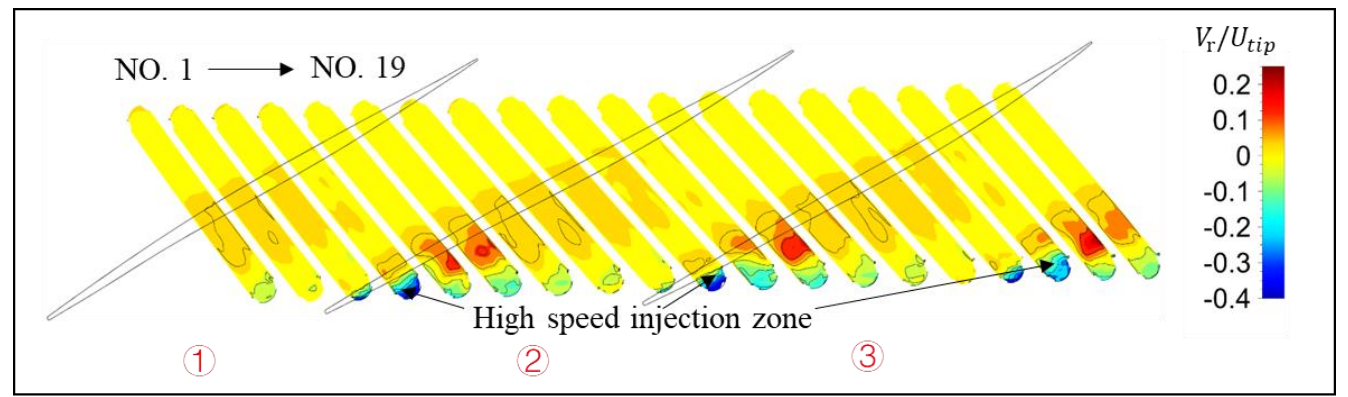

(b) $80 \%$ design speed

Fig. 15. Radial velocity distribution on the slot opening surface of SRSCT.

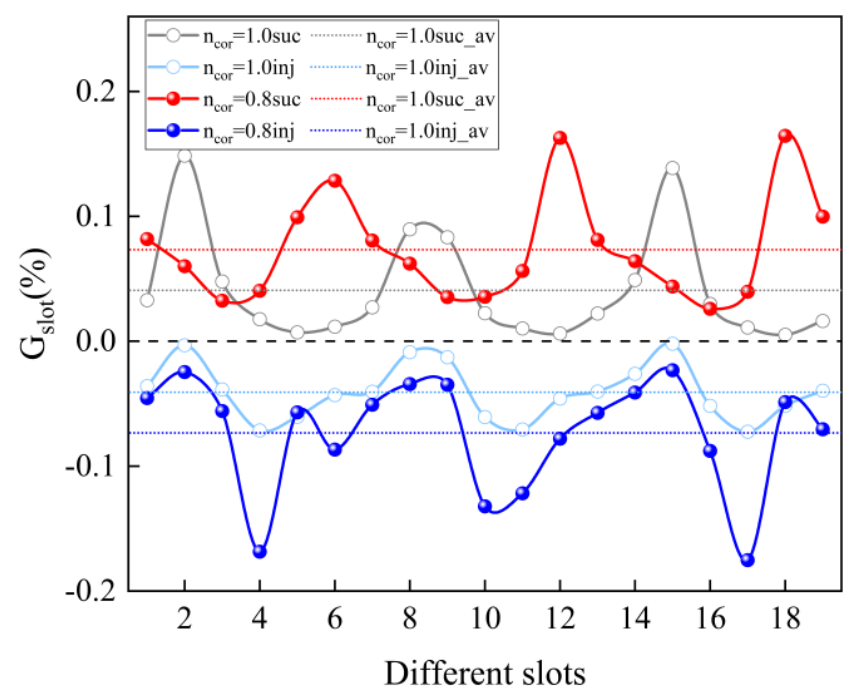

Fig. 16. Normalized mass flow distributions through opening surface of different slots.

(identified by $n_{\text {cor }}=1.0 \mathrm{suc}, n_{\text {cor }}=1.0 \mathrm{inj}$ etc.) represent the suction mass flow and injection mass flow at different speeds, and the horizontal dotted line with different color (identified by $n_{\text {cor }}=1.0$ suc_av, $n_{\text {cor }}=1.0$ inj_av etc.) present the average value of suction/injection mass flow.

The effect of strong shock wave on slot flow recirculation is more intuitive based on mass flow distribution at different rotational speeds. Compared with the existence of separate suction effect slots (the instantaneous injection mass flow is zero) due to strong shock wave at $100 \%$ design speed, more effective slot circulation can be observed at $80 \%$ design speed.

\subsection{Instability characteristics under SRSCT}

Although the SRSCT improves the tip blockage of compressor rotor and brings about the stability enhancement of compressor, the influence of CT on rear stator is often overlooked and rarely discussed. In this paper, we try to explain the instability characteristics under the effect of SRSCT, so some quantified parameters and visualized flow fields of stator passage are analyzed at CTNS condition. Figure 17 demonstrates the changes of mass flow in the meridian plane for CTNS1 and CTNS2 (which can be found in Fig.5) at $80 \%$ design speed, the changed value of mass flow is the difference between 

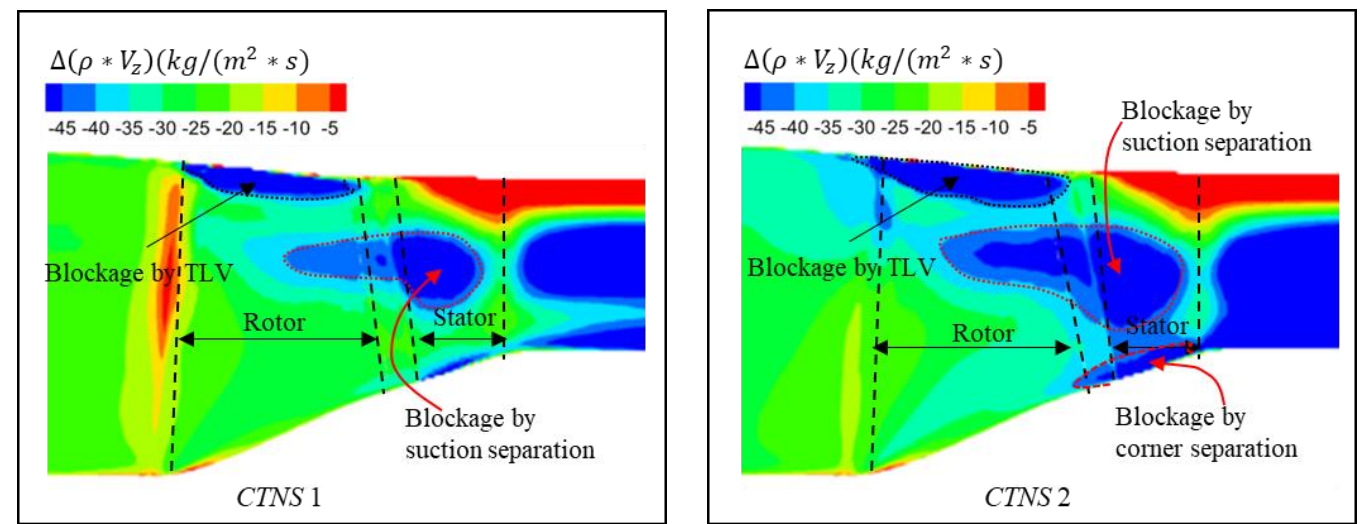

Fig. 17. Mass flow distribution in the meridian plane at two different conditions at $\mathbf{n}_{\text {cor }}=0.8$.

CTNS points and peak efficiency point. With the mass flow approaching instability boundary, for example, from the CTNS1 condition to CTNS2 condition, the blockage induced by tip leakage vortex slightly increases, while the blockage caused by stator suction surface separation and stator corner separation is significantly larger. So we can deduce that the stator passage blockage or corner separation may be dominant for inducing the compressor stage with SRSCT into unstable flow condition.

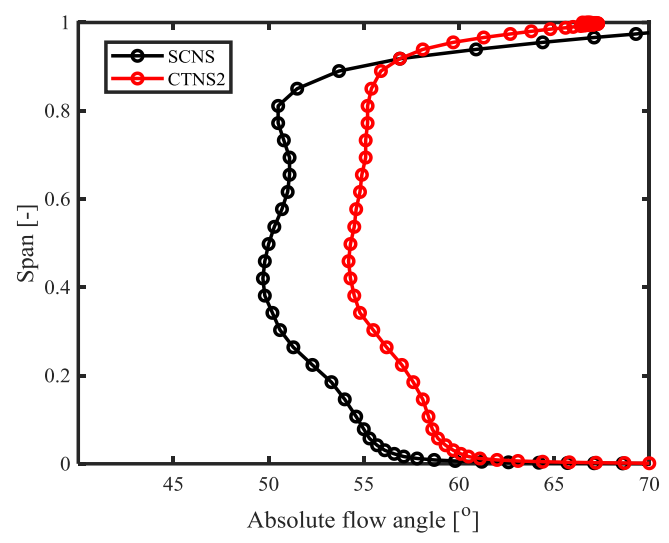

Fig. 18. Radial distributions of absolute stator inflow angle at $\mathbf{n}_{\text {cor }}=0.8$.
In order to further analyze the evolution mechanism of the blockage in the stator passage, the timeaveraged and pitch-averaged radial distributions of absolute stator inflow angle at SCNS condition and CTNS condition at $80 \%$ design speed are demonstrated in Fig.18. Compared to SCNS condition, absolute stator inflow angle increases by 5 8 degrees on average below 0.9 span at CTNS2 condition. As a result, the stator must work at higher attack angle, which will cause vast stator suction surface separation or stator corner separation. Finally, the stator blockage stall may occur if the stator inflow angle exceeds the limit of attack angle.

Based on the above analysis, Fig. 19 shows the transient 3D streamline distributions in stator passages at CTNS2 condition at two different moments $\mathrm{T} 1$ and $\mathrm{T} 2$, where the number (1) to (7) represent different stator passages. It demonstrates that large shedding vortex appears in stator passage (2) and (5) at moment T1, while vanishes at moment T2. At the meantime, large shedding vortex appears at stator passage (1) and (7). The unsteady characteristics of stator passage blockage are easy to arise the circumferential stacking of blockage and induce the stator stall cells, which may trigger the compressor into instability flow condition. It should be noted that the effect of SRSCT on the unsteady characteristics of stator passage vortex is worthy of making a profound study, and it will be analyzed in subsequent studies.

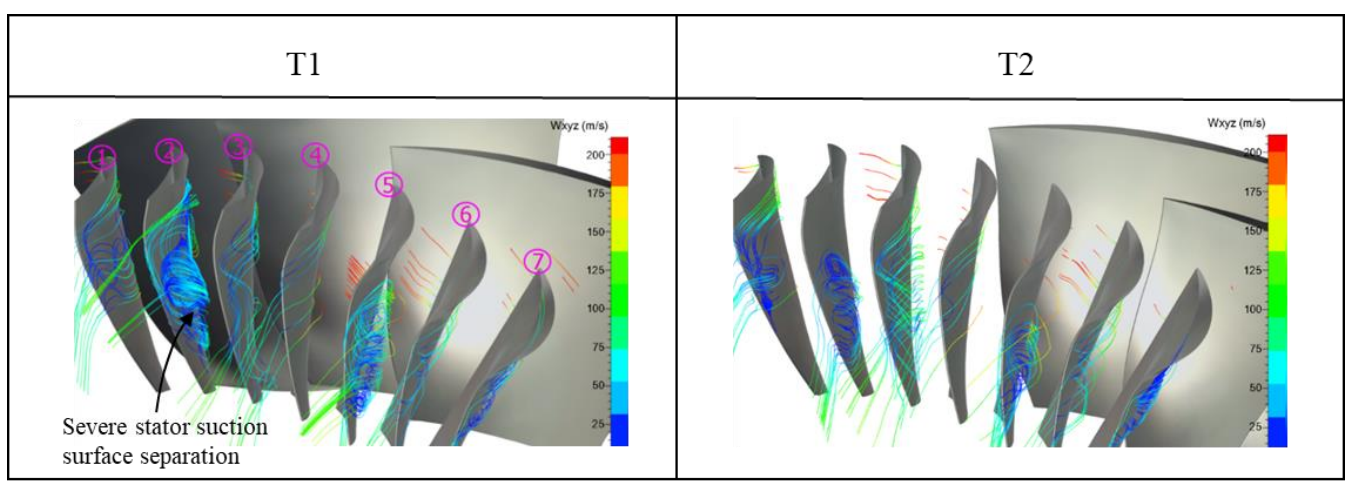

Fig. 19. Instantaneous 3D streamline distributions in stator passages at CTNS2 at $80 \%$ design speed. 


\section{CONCLUSIONS}

In this paper, a novel shallow reversed slot-type casing treatment (SRSCT) is proposed and analyzed in a transonic compressor stage numerically. The conclusions can be summarized as follows:

(1) In investigated compressor, the stall mechanism is different at two rotational speeds. At design speed, blade tip stall may occur because of suction surface separation, yet the blockage induced by TLV dominates at off-design speed. The results demonstrate the difference of stall margin improvement at different speeds result from the difference of dominant factors causing tip blockage. The SRSCT is more effective when tip blockage is dominated by TLV. Meanwhile, the intensity or extent of tip loading reduction and mass flux redistribution can be an indicator of effectiveness of SRSCT.

(2) Through the analysis on the influence of passage shock on the effectiveness of SRSCT, it is recommended that a suitable position must be fully considered to ensure the effective flow recirculation in slots. At design speed, a fraction of invalid slot flow recirculation happens due to strong passage shock, which leads to the insufficient stall margin improvement with SRSCT. At off-design speed, the shock wave is entirely detached and the intensity of shock wave weakens, so the invalid flow recirculation caused by strong shock wave gets relief.

(3) The analysis of instability characteristics of compressor with SRSCT shows that stator passage blockage tends to trigger the compressor into stall. As the mass flow approaching the instability boundary, the rear stator may deviate from the design point due to the effect of casing treatment, which will inevitably result in reduced effectiveness of casing treatment and increased flow losses. Therefore, the problem of stage matching caused by applying $C T$ will be studied in the future.

\section{ACKNOWLEDGEMENTS}

The authors would like to thank the support of the National Science and Technology Major Project (No. 2017-II-0005-0018), National Natural Science Foundations (Grants Nos. 51536006, 51006084 and 51576162) are also appreciated.

\section{REFERENCES}

Alone, D. B., S. S. Kumar, S. M. Thimmaiah and A. M. Pradeep (2016). Stability management of high speed axial flow compressor stage through axial extensions of bend skewed casing treatment. Propulsion and Power Research 5(3), 236-249.

Brandstetter, C., F. Wartzek, J. Werner, H. P. Schiffer and F. Heinichen (2015). Unsteady measurements of periodic effects in a transonic compressor with casing treatments. In ASME Turbo Expo 2015: Turbine Technical Conference and Exposition, June 15-19, Montreal, Canada.

Chima, R. V. (2009). Swift code assessment for two similar transonic compressors. AIAA paper, 2009-1058.

Day, I. J. (2016). Stall, surge and 75 years of research. Journal of Turbomachinery 138(1):116.

$\mathrm{Du}$, J. and J. R. Seume (2017). Design of casing treatment on a mixed-flow compressor. ASME Turbo Expo 2017: Turbomachinery Technical Conference and Exposition.

Donald, S. Madden and Matt A. West (2005). Effects of inlet distortion on the stability of an advanced military swept fan stage with casing treatment. In ASME turbo expo 2005: Power for Land, Sea and Air, June 6-9, 2005, Reno-Tahoe, Nevada, USA.

Fujita, H. and H. Takata (1984). A study on configurations of casing treatment for axial flow compressors. Bulletin of JSME, 27(230), 1675-1681.

Furukawa, M., M. Inoue, K. Saiki and K. Yamada (1998). The role of tip leakage vortex breakdown in compressor rotor aerodynamics, ASME Paper 98-GT-239.

Greitzer, E. M., J. P. Nikkanen and D. E. Haddad (1979). A fundamental criterion for the application of rotor casing treatment. Journal of Fluids Engineering 101, 237-243.

Gelmedov, F. S., V. I. Mileshin and P. G. Kozhemyako (2014). Stall margin improvement in three-stage low pressure compressor by use of slot type casing treatments. ASME paper GT 2014-26298.

Hathaway, M. D. (2002). Self-recirculating casing treatment concept for enhanced compressor performance. ASME GT 2002-30368.

Heinrich, M., H. Khaleghi and C. Friebe (2020). Effect of Circumferential Casing Treatment on Low-Speed Contra-Rotating Fans. Journal of Applied Fluid Mechanics 13(6), 1719-1726.

Hembera, M., H. P. Kau and E. Johann (2008). Simulation of casing treatments of a transonic compressor stage. International Journal of Rotating Machinery 44(47), 8605-8607.

Hofmann, W. and J. Ballmann (2002). Tip clearance vortex development and shock-vortexinteraction in a transonic axial compressor rotor, AIAA Paper 2002-0083.

Houghton, T. and I. J. Day (2012). Stability enhancement by casing grooves: the importance of stall inception mechanism and solidity. Journal of Turbomachinery 134(2), 1-8.

Koch, C. C. (1970). Experimental evaluation of outer 
Z. D. Chi et al. / JAFM, Vol. 14, No. 6, pp. 1691-1704, 2021.

case blowing or bleeding of single stage axial flow compressor, part VI: Final report.

Li, J., J. Du and C. Nie (2019). Review of tip air injection to improve stall margin in axial compressors. Progress in Aerospace Sciences, 106(APR), 15-31.

Lu, X., W. Chu and J. Zhu (2006). Experimental and numerical investigation of a subsonic compressor with bend skewed slot casing treatment. ASME Turbo Expo 2006: Power for Land, Sea, and Air.

NUMECA Inc (2011). NUMECA Theoretical Manual-FINE Turbo. Brussels, BE.

Reid, L. and R. D. Moore (1978). Design and overall performance of four highly loaded, high speed inlet stages for an advanced high-pressure-ratio core compressor. NASA Technical Paper, NASA-TP-1337.

Streit, J. A., C. Brandstetter and F. Heinichen (2013). An advanced axial-slot casing treatment on a transonic compressor: A close look with computational fluid dynamics and experimental validation. Proceedings of the Institution of Mechanical Engineers, Part A: Journal of Power and Energy 227(6), 683-691.

Suder, K. L. and M. D. Hathaway (2001). Compressor stability enhancement using discrete tip injection. Journal of Turbomachinery 123(1), 14-23.

Sun, X., X. Dong and D. Sun (2019). Recent development of casing treatments for aeroengine compressors. Chinese Journal of
Aeronautics 32(01), 4-39.

Vo, H. D. (2008). Criteria for Spike Initiated Rotating Stall. Proceedings of Asme Turbo Expo 130 (1), 155-165.

Wilke, I. and H. P. Kau (2004). A numerical investigation of the flow mechanisms in a high pressure compressor front stage with axial slots. Journal of Turbomachinery, Vol. 126.

Wang, W., J. Lu, X. Luo, R. Huang and W. Chu (2020). Failure mechanism of casing treatment in improving stability of a highly loaded twostage axial compressor, Aerospace Science and Technology, 105, 105979.

Wang, W., J. Lu, X. Luo and W. Chu (2019). Coupling method of stability enhancement based on casing treatments in an axial compressor. Aerospace Science and Technology, 95 (105449).

Wilke, I., H. P. Kau and G. Brignole (2005). Numerically aided design of a high-efficient casing treatment for a transonic compressor. ASME GT 2005-68993.

Zhang, H., W. Liu, E. Wang, W. Chu, J. Yang and W. Zhao (2020). Numerical Investigation of the Influence of Axial Overlap of Blade Angle Slots on an Axial Flow Compressor Stability. Journal of Applied Fluid Mechanics 13(6), 1693-1705.

Zhou, X., Q. Zhao, W. Cui and J. Xu (2017). Investigation on axial effect of slot casing treatment in a transonic compressor. Applied Thermal Engineering, 126, 53-69. 